\title{
ON THE ZEROS OF THE DERIVATIVES OF A FUNCTION AND ITS ANALYTIC CHARACTER
}

\author{
G. POLYA
}

1. Introduction. How do the zeros of the $n$th derivative $f^{(n)}(x)$ behave when $n$ becomes very large? How does this behavior depend on the analytic nature of the function $f(x)$ ? At first sight this question appears to be a little out of the way. In fact it is intimately connected with essential parts of the theory of functions. In the Hadamard theory of the singularities of the Taylor series we consider the sequence of the derivatives $f(x), f^{\prime}(x), f^{\prime \prime}(x), \cdots$ for a fixed complex value of $x$; in the theory of quasi-analytic functions we consider the same sequence for variable real $x$, and we consider especially the maximum absolute value of each of its terms in a given interval; now we consider again the same sequence $f(x), f^{\prime}(x), f^{\prime \prime}(x), \cdots$ for variable $x$, that may be complex or real, and we consider especially the values of $x$ for which its terms vanish. In all three cases the main object of consideration is the connection of the chosen feature of the sequence $f(x), f^{\prime}(x), f^{\prime \prime}(x), \cdots$ with the analytic nature of the function $f(x)$.

There is a special reason for giving a general survey of results about the zeros of successive derivatives just now. The subject received quite recently essential contributions from several mathematicians in this country, from R. P. Boas, Einar Hille, A. C. Schaeffer, I. J. Schoenberg, Gabor Szegö, J. D. Tamarkin, D. V. Widder, Norbert Wiener and the present speaker. All these contributions are linked together and seem to open the door to further results and to a well connected harmonious theory.

In this short talk I should like to emphasize the main outline and the essential divisions of the theory in so far as they are recognizable today. In order to do this as clearly and intuitively as I can, I have to discuss some of the older results, to leave out some of the newer ones and to sacrifice precise details except for a few central points.

Our subject has two main parts. In one we consider analytic functions of a complex variable, in the other real-valued functions of a real variable. I proceed to describe these two parts in this order.

An address presented before the New York meeting of the Society on April 3, 1942, by invitation of the Program Committee; received by the editors May 8, 1942. 


\section{THE COMPLEX DOMAIN}

2. Statement of the problem. In the next sections (2 to 7$) f(z)$ denotes an analytic function of the complex variable $z$. Let us assume that $f(z)$ is single-valued and analytic in the whole plane, except maybe in certain isolated singular points.

Let us consider the zeros of $f(z)$; they form a more or less irregular distribution of points in the plane. Now let us pass to the derivative $f^{\prime}(z)$ and consider its zeros; they are in general different from those of $f(z)$ and form a new distribution of points. If we pass, by differentiating once more, to $f^{\prime \prime}(z)$ and its zeros, the picture changes again, the points appear to have moved into a third position. Corresponding to the sequence of the derivatives $f(z), f^{\prime}(z), f^{\prime \prime}(z), \ldots$ we have a sequence of distributions of points formed by the zeros of the successive derivatives. Can we discover in this sequence of pointdistributions some definite trend?

It is not bad to start from a vague question that is intuitive and suggestive, provided that it leads us finally to some precise question. What precise question is suggested by the intuitive process we are considering, the migration of zeros in the course of successive differentiations?

In fact, not only one question, but several different precise questions can be put. But we need a few definitions.

(I) Let us consider any fixed regular point of $f(z)$; let us assume, to be concrete, that $z=0$ is a regular point. Let $r_{n}$ denote the absolute value of the zero of $f^{(n)}(z)$ which is nearest to the origin.

(II) Let us consider any fixed closed circle in which $f(z)$ is regular. Let us assume that $f(z)$ is regular in the closed unit circle $|z| \leqq 1$, and let $N_{n}$ denote the number of zeros of $f^{(n)}(z)$ (with multiplicity) in this circle.

(III) We define a set $D^{\prime}$, attached to the function $f(z)$, in the following way. Consider a point $z$ of the plane. If there exists a circle of center $z$ in which only a finite number of derivatives vanishes, $z$ does not belong to $D^{\prime}$. In the other case, when any (arbitrarily small!) fixed circle with center $z$ contains the zeros of an infinity of derivatives $f^{\left(n_{1}\right)}(z), f^{\left(n_{2}\right)}(z), \cdots, z$ belongs to $D^{\prime}$. The set $D^{\prime}$ is in some sense the final position of the zeros of $f^{(n)}(z)$; let us call $D^{\prime}$ the final set of $f(z)$. If $f(z)$ is meromorphic or entire, the final set $D^{\prime}$ can also be described as the derived set of the denumerable set $D$ of all zeros of all derivatives $f(z), f^{\prime}(z), f^{\prime \prime}(z), \cdots$ (provided that points in which an infinity of derivatives vanishes are also counted as points of $D^{\prime}$ ).

How do the zeros of the derivatives approach a regular point? What can we say about the sequence $r_{1}, r_{2}, r_{3}, \ldots$ ? Do the zeros of 
$f^{(n)}(z)$ become denser as $n$ increases? What about the sequence $N_{1}, N_{2}, N_{3}, \cdots$ ? Does the configuration of the zeros of $f^{(n)}(z)$ tend to a final position? Can we find the final set $D^{\prime}$ ?

3. Meromorphic functions. The answer to all these questions depends on the analytic character of the function $f(z)$. For functions of certain sorts we can discover a trend, and this trend is particularly easy to recognize when $f(z)$ is a meromorphic function. In this case the trend may be roughly described by saying that the poles of $f(z)$ act as repulsive centers on the zeros of $f^{(n)}(z)$.

We can give a precise description after a precise definition. Those points of the plane which are nearer to a pole $a$ of $f(z)$ than to any other pole of $f(z)$ constitute the domain of the pole $a$. If the domains of two poles, $a$ and $b$, have common boundary points, these are on the perpendicular bisector of the line that joins $a$ to $b$. Therefore the boundary of the domain of the pole $a$ is a polygonal line, and the domain itself is an open convex set. The zeros of $f^{(n)}(z)$ are pushed toward the polygonal boundary of the domain of the pole as $n$ increases. The final set of a meromorphic function contains no point interior to the domain of any pole but contains all points which are on the common boundary of the domains of two or more poles [20].

Observe that the final set depends only on the position of the poles of $f(z)$, but is independent of the multiplicity of these poles, or their residues, and so on.

4. Functions with an essential singularity. Let us consider next the case in which $f(z)$ has just one finite singular point, the point $z=0$, say. If this point is a pole the whole plane is its domain, the zeros of $f^{(n)}(z)$ are pushed towards $\infty$, they have no finite limiting point, the final set is empty. But if the point $z=0$ is an essential singular point, the situation is quite different; the trend of the movement of the zeros of $f^{(n)}(z)$ with increasing $n$ is much more difficult to recognize, and it is no longer true that this trend depends only on the position of the singular point. For example, if

$$
f(z)=(1 / z) e^{-1 / z},
$$

all zeros of $f^{(n)}(z)$ are positive and real and the final set is the positive half of the real axis. If we change $z$ into $z e^{i \alpha}$, where $\alpha$ is a fixed real number, $0<\alpha<2 \pi$, the singularity of $f(z)$ remains at the point 0 , but the zeros of $f^{(n)}(z)$ tend to condense along another ray issued from the singular point; the final set changes position.

5. Entire functions. Let us now consider a function with no finite 
singular points, an entire function. Can we recognize the trend of the zeros of $f^{(n)}(z)$ as $n \rightarrow \infty$ ? We may say that this trend depends mainly on the rate at which $f(z)$ increases when $|z| \rightarrow \infty$, that is, on the order of $f(z)$. For certain entire functions of order 1 , as, for example, for

$$
f(z)=\sin z
$$

the differentiation does not change essentially the density of the distribution of the zeros. If the order of $f(z)$ is less than 1 the differentiation tends to scatter the zeros; the zeros of $f^{(n)}(z)$ tend to move out to $\infty$ as $n$ increases; their distribution becomes thinner. If the order of $f(z)$ is greater than 1 the differentiation tends to concentrate the zeros; the zeros of $f(z)$ tend to move in from $\infty$ as $n$ increases; their distribution becomes denser. In short, the point at $\infty$ exerts on the zeros of $f^{(n)}(z)$ attraction or repulsion, according as the order of $f(z)$ is less than 1 or greater than 1 .

Passing to precise statements, if $\lambda$ denotes the order of the entire function $f(z)$, we have

$$
\begin{aligned}
& \limsup (\log n)^{-1} \log r_{n} \geqq(1-\lambda) / \lambda, \\
& \liminf _{n \rightarrow \infty}(\log n)^{-1} \log N_{n} \leqq(\lambda-1) / \lambda .
\end{aligned}
$$

The inequality (1) was found, at least for $\lambda<1$, by Ålander [1]. More relations and more precise relations of this kind were discovered by Gontcharoff and several authors following his line of research. ${ }^{1}$ The inequality (2) seems to be new. Both inequalities are "exact," that is, the sign of equality may be attained for certain special functions. Furthermore we cannot exchange lim inf and lim sup or change either into lim without falling into false assertions. ${ }^{2}$

6. Real entire functions. An entire function $f(z)$ is called real if it takes real values for real $z$, or, what is the same, if the coefficients of its Maclaurin series are all real. The set of zeros of a real entire function is symmetrical with respect to the real axis. The real axis seems to exert an influence on the complex zeros of $f^{(n)}(z)$; it seems to at-

\footnotetext{
${ }^{1}$ Gontcharoff [14]. See also Takeneka [30-34], Kakeya [16], Whittaker [35, 36], Schoenberg [28]. Boas [9] (see also Levinson [17]) introduced a quantity related to but different from $r_{n}$; let $s_{n}$ denote the radius of the largest circle of center $z=0$ in the interior of which $f(z)$ is regular and $f^{(n-1)}(z)$ is univalent. Visibly $r_{n} \geqq s_{n}$. It seems that $s_{n}$ has more simple properties than $r_{n}$; especially we have the following double inequality $\lim \inf _{n \rightarrow \infty}(\log n)^{-1} \log s_{n} \leqq(1-\lambda) / \lambda \leqq \lim \sup _{n \rightarrow \infty}(\log n)^{-1} \log s_{n}$.

${ }^{2}$ If $\lambda \leqq 1$ the final set may be empty and we may construct entire functions of any given order whose final set consists of just one point. If $\lambda>1$, must the final set necessarily contain a point?
} 
tract these zeros when the order is less than 2 , and it seems to repel them when the order is greater than 2 .

We may pass to exact statements, but these are, for the time being, hypothetical.

A. If the order of the real entire function $f(z)$ is less than 2 , and $f(z)$ has only a finite number of complex zeros, then its derivatives, from a certain one onwards, will have no complex zeros at all.

B. If the order of the real entire function $f(z)$ is greater than 2, and $f(z)$ has only a finite number of complex zeros, then the number of the complex zeros of $f^{(n)}(z)$ tends to infinity as $n \rightarrow \infty$.

Both theorems are hypothetical, but $\mathrm{A}$ is better known than B. A weaker theorem than $A$, with $2 / 3$ instead of 2 , was first proved, then $2 / 3$ was replaced by 1 , and at last by $4 / 3 .^{3}$ So we know today that real entire functions of order less than $4 / 3$ lose all their complex zeros after a finite number of differentiations provided they had only a finite number to start with. But the advance from $4 / 3$ to 2 , that is, to the full theorem $A$, is still to be made.

B is supported mostly by "experimental" evidence. ${ }^{4}$ I quote two interesting examples

$$
e^{-z^{2 k}}, \quad e^{-e^{z} z}
$$

The final set of the first entire function (3) ( $k$ is a positive integer) consists of $k$ straight lines passing through the origin and dividing the plane into $2 k$ equal angles [20]. The final set of the second entire function (3) consists of an infinity of parallel lines, dividing the plane into congruent strips of width $2 \pi .^{5}$ The final set of both functions contains the whole real axis, but also points outside the real axis (provided $k \geqq 2$ ).

7. Another open question about real entire functions. The consider-

${ }^{3}$ The hypothetical theorem A (or rather a slightly more general theorem in which certain entire functions of order 2 are also considered) was found independently by two authors, A. Wiman and G. Polya. The theorem with $2 / 3$ was stated as proved by Polya [21, see p. 27, annotation 21]; proved, with reference to this first statement, almost simultaneously by Ålander [4] and Polya [22]. The theorem with 1 was proved by Wiman [39], that with $4 / 3$ by Polya [23]. See also Wiman [38].

${ }^{4} \mathrm{~A}$ first remark in the direction of theorem $\mathrm{B}$ was made by Polya [18, 19]. (The role of the order 2 is indicated by the following theorem, due to Laguerre. $A$ real entire function that has only real zeros is or is not a limit of polynomials that have only real zeros according as its order is less than 2 or greater than 2.) A more precise hypothetical theorem was formulated by A. Wiman and investigated by Ålander [1, 2] who used a geometrical method.

${ }^{6}$ Suggested by the present speaker, proved by G. Szegö (unpublished). 
tion of the general trend of the zeros of $f^{(n)}(z)$ and some examples, principally (3), may lead us to the following hypothetical theorem.

C. If a real entire function of order greater than 1 remains bounded for real values of the variable, then its final set contains the whole real axis.

I state this theorem without any pretension to the gift of prophecy. I state it because I think that the problem to prove or to disprove it, is neither too trivial nor completely inaccessible.

\section{THE REAL DOMAIN}

8. Notation and introductory results. We change our notation. Henceforth $f(x)$ denotes a real-valued function of the real variable $x$, that is defined and possesses derivatives of all orders in a certain interval $I$, and $N_{n}$ denotes the number of changes of sign of $f^{(n)}(x)$ in $I$.

$f(x)$ need not be analytic in $I$. The analyticity of $f(x)$ is bound to the condition that to each point $a$ of $I$ corresponds a positive $\rho=\rho(a)$, such that, in the common part of the two intervals $I$ and $(a-\rho, a+\rho)$, $f(x)$ is represented by a convergent power series in $(x-a)$. If $f(x)$ is analytic in $I$, its analytic continuation is completely determined by its values at the points of $I$ and, if the continuation exists, it may turn out that $f(x)$ is meromorphic, or entire, or entire of finite order, and so on.

The number $N_{n}$ may be infinite. But if the interval $I$ is closed, and $f(x)$ is analytic in $I$, not only is $N_{n}$ a finite number for each $n$ but the increase of $N_{n}$ with $n$ is limited by the following theorem.

If $f(x)$ is analytic in the closed interval $I$, then

$$
\lim _{n \rightarrow \infty} \inf n^{-1} N_{n} \text { is finite. }
$$

If $f(x)$ is an entire function

$$
\lim _{n \rightarrow \infty} \inf n^{-1} N_{n}=0 .
$$

\section{If $f(x)$ is an entire function of the finite order $\lambda$}

$$
\lim \inf (\log n)^{-1} \log N_{n} \leqq(\lambda-1) / \lambda .
$$

If $f(x)$ is an entire function of exponential type (that is, if its increase does not exceed the finite type of order 1)

$$
\lim _{n \rightarrow \infty} \inf N_{n} \text { is finite. }
$$

The letters a, e, $\mathrm{f}, \mathrm{t}$ will be used systematically in distinguishing formulas, a reminding us of analytic, e of entire, $f$ of finite order, $t$ of 
exponential type. Observe that the increase of $N_{n}$ is more and more restricted as we pass along the cases a, e, f, $t$.

The theorem we just stated starts from a given analytic character of the function (as analytic, entire and so on) and reaches conclusions concerning the zeros of the derivatives, especially conclusions concerning the behavior of the sequence $N_{1}, N_{2}, N_{3}, \cdots$; the theorem seems to be new, it is easy to prove by "complex variable" methods, and appropriate to introduce our subject. We pass now to results of a different character in which we start from the derivatives, especially from properties of the sequence $N_{1}, N_{2}, N_{3}, \cdots$, and reach conclusions about the analytic nature of the function, using mainly "real variable" methods.

9. All derivatives are of constant sign. The first relationships between the analytic character of a real function and the qualitative behavior of its derivatives were discovered by S. Bernstein. One of his simplest results is concerned with the condition

$$
N_{n}=0, \quad n=0,1,2, \cdots,
$$

and may be stated as follows.

If no derivative of $f(x)$ changes sign in an open interval $I$, then $f(x)$ is analytic in $I$.

This result came as a great surprise at the time of its first publication [5], and it still occupies a central position. It is the common starting point of many questions and generalizations.

10. An infinity of derivatives is of constant sign. A first generalization of the condition (5) is due to S. Bernstein himself [5].

If infinitely many derivatives $f^{\left(n_{1}\right)}, f^{\left(n_{2}\right)}, \cdots$ are of constant sign in the open interval $I$,

$$
N_{n k}=0, \quad k=1,2,3, \cdots
$$

and if the sequence $n_{1}, n_{2}, \cdots$ does not increase more rapidly than a geometric progression, that is, if there is a fixed quantity $Q$ such that

$$
n_{k+1} / n_{k}<Q, \quad k=1,2,3, \cdots,
$$

then it still may be asserted that $f(x)$ is analytic in $I$.

Recently R. P. Boas gave a new proof of this result and showed by interesting examples that the condition expressed by (6) and (7) cannot be essentially relaxed without endangering the truth of the theorem [10].

11. All derivatives are of constant sign. Influence of the signs. 
Let us return to the central condition (5). If it holds, each derivative of $f(x)$ has a constant sign in the open interval $I$, and, therefore, each derivative is monotonic in $I$, and so is the absolute value of each derivative. In fact, since

$$
(d / d x)\left[f^{(n)}(x)\right]^{2}=2 f^{(n)}(x) f^{(n+1)}(x)
$$

the absolute value of $f^{(n)}$ is steadily increasing or steadily decreasing, according as $f^{(n)}$ and $f^{(n+1)}$ are or are not of the same sign. We say that $f^{(m)}$ and $f^{(n)}$, where $m<n$, belong to the same block if and only if the absolute values of $f^{(m)}, f^{(m+1)}, \cdots, f^{(n-1)}, f^{(n)}$ all vary in the same sense, that is, all increase or all decrease. Thus $f^{(n)}$ and $f^{(n+1)}$ belong to different blocks if and only if

$$
f^{(n)}(x) f^{(n+2)}(x)<0
$$

in $I$. Let $l_{1}, l_{2}, l_{3}, \cdots$ denote the lengths of the successive blocks into which the sequence $f, f^{\prime}, f^{\prime \prime}, \ldots$ is decomposed; we assume here that no block has infinite length, and that therefore there is an infinity of blocks. S. Bernstein discovered that the lengths of these blocks are connected with the analytic nature of $f$. He proved the following theorem. ${ }^{6}$

If, in the open interval $I$,

$$
l_{n}=o\left(l_{1}+l_{2}+\cdots+l_{n}\right)
$$

$f(x)$ is an entire function. If there exists a positive number $\lambda, \lambda>1$, such that

$$
l_{n}=O\left(\left[l_{1}+l_{2}+\cdots+l_{n}\right]^{(\lambda-1) / \lambda}\right)
$$

$f(x)$ is an entire function of finite order not exceeding $\lambda$. If

$$
l_{n}=O(1)
$$

$f(x)$ is an entire function of exponential type.

12. An infinity of derivatives is of constant sign. Influence of the signs. D. V. Widder found recently [37] that $f(x)$ is necessarily an entire function of exponential type if, in $I$,

$$
(-1)^{m} f^{(2 m)}(x) \geqq 0, \quad m=0,1,2, \cdots .
$$

This condition would imply, if we knew that no derivative of $f(x)$ vanishes in $I$, that $f^{(2 m)}$ and $f^{(2 m+1)}$ belong to different blocks, and

${ }^{6}$ Bernstein's statement of his results $[7,8]$ is different in expression but not in substance from the statement given here. 
that, therefore, no block has a length greater than two, $l_{k} \leqq 2$. But, in fact, Widder's condition (8) does not say anything about the nonvanishing of the derivatives of odd order in $I$, and, therefore, Widder's theorem is not contained in the third part of Bernstein's theorem stated in the foregoing section; (8), although of much more special nature, does not imply $(7 \mathrm{t})$.

R. P. Boas and the present speaker set out to find a common generalization of S. Bernstein's result, stated in the foregoing section, and of Widder's result, quoted in the present section. They obtained the following theorem $[12,13]$.

Let $\left\{n_{k}\right\}$ and $\left\{q_{k}\right\}$ be sequences of positive integers, $\left\{n_{k}\right\}$ strictly increasing. Assume that $f^{\left(n_{k}\right)}$ and $f^{\left(n_{k+2} q_{k}\right)}$ do not change sign in $I$,

$$
N_{n k}=N_{n k+2 q_{k}}=0, \quad k=1,2,3, \cdots,
$$

that

in $I$, and that

$$
f^{\left(n_{k}\right)}(x) f^{\left(n_{k}+2 q_{k}\right)}(x) \leqq 0
$$

If

$$
q_{1}+q_{2}+\cdots+q_{k}=O\left(n_{k}\right)
$$

then $f(x)$ is an entire function. If there is a positive number $\lambda, \lambda>1$, such that

$$
n_{k}-n_{k-1}=O\left(n_{k}^{(\lambda-1) / \lambda}\right), \quad q_{k}=O\left(n_{k}^{(\lambda-1) / \lambda}\right),
$$

then $f(x)$ is an entire function of finite order not exceeding $\lambda$. If

$$
n_{k}-n_{k-1}=O(1), \quad q_{k}=O(1),
$$

then $f(x)$ is an entire function of exponential type.

This theorem is more general than that of Bernstein quoted in the foregoing section, since the condition (9) is less restrictive than (5). We derive Bernstein's theorem from the present one by putting $l_{1}+l_{2}+\cdots+l_{k}=n_{k}$ and $q_{k}=1$, for $k=1,2,3, \cdots$. We obtain Widder's result, quoted in this section, by putting $n_{k}=2 k$ and $q_{k}=1$.

13. No derivative changes sign more than a given number of times. The case of periodic functions. Let us return again to the fundamental theorem considered in $\$ 9$. S. Bernstein derived the analyticity of the function from the condition (5). Is it possible to derive analyticity from the less restrictive condition

$$
N_{n}=O(1),
$$


that is, does the condition that no derivative changes sign more than a fixed number of times imply the analyticity of the function? This question was first answered in the special case of periodic functions by Norbert Wiener and the present speaker [25], and led to the following precise result.

If no derivative of the periodic function $f(x)$ of period $2 \pi$ has more than $2 m$ changes of sign in a period, $f(x)$ is a trigonometric polynomial of order not exceeding $m$,

$$
f(x)=a_{0}+a_{1} \cos x+b_{1} \sin x+\cdots+a_{m} \cos m x+b_{m} \sin m x
$$

with certain constants $a_{0}, a_{1}, b_{1}, \cdots, a_{m}, b_{m}$.

Any derivative of a trigonometric polynomial of order $m$ is a trigonometric polynomial of the same order and has no more than $2 m$ real zeros in a period. But if a periodic function is not a trigonometric polynomial the number of changes of sign of the $n$th derivative must tend to infinity with $n$; this is contained in the theorem. We can restate the theorem by saying that the number of changes of sign of the $n$th derivative of a periodic function does or does not tend to infinity, according as its Fourier series has or has not an infinity of coefficients different from zero. Thus the theorem discloses a new characteristic property of trigonometric polynomials, the uniform boundedness of the changes of sign of all derivatives.

14. The number of changes of sign of the $n$th derivative has a prescribed bound depending on $n$. The case of periodic functions. The theorem of the preceding section became a starting point of new questions and new generalizations. A first generalization may be formulated as the following tripartite theorem.

Let $f(x)$ denote a periodic function and $N_{n}$ the number of changes of sign of $f^{(n)}(x)$ in a period. If

$$
N_{n}<2 n / \log n
$$

for sufficiently large $n$, then $f(x)$ is an entire function. If there is a $\lambda$ $(\lambda>1)$ such that

$$
\lim \sup (\log n)^{-1} \log N_{n} \leqq(\lambda-1) / \lambda,
$$

$f(x)$ is an entire function of finite order not exceeding $\lambda$. If

$$
N_{n}=O(1)
$$

$f(x)$ is an entire function of exponential type.

A similar but less precise tripartite statement was obtained by Norbert Wiener and the present speaker [25]. The exact form just 
stated is due to Gabor Szegö [29]. As it may be shown by suitable examples, the condition (11f) is completely sharp, and (11e) differs very little (if it differs at all) from a completely sharp condition.

The condition (11t) reproduces (11), and the third part of the theorem is identical with the theorem of the foregoing section; in fact, as is well known, a periodic entire function of exponential type is just a trigonometric polynomial. The conditions (11e), (11f), (11t) are parallel to the foregoing series of conditions $(4 \mathrm{e}),(4 \mathrm{f}),(4 \mathrm{t}) ;(7 \mathrm{e}),(7 \mathrm{f}),(7 \mathrm{t})$; and $(10 \mathrm{e}),(10 \mathrm{f}),(10 \mathrm{t})$. The hypotheses $(11 \mathrm{e}),(11 \mathrm{f}),(11 \mathrm{t})$ restrict more and more the increase of $N_{n}$; the last one does not allow any "increase" at all we may say. The corresponding conclusions (that $f(x)$ is entire, entire of finite order, entire of exponential type) may be expressed by a more and more rapid decrease of the coefficients of the Fourier series; in the last case the decrease is so abrupt that all coefficients are equal to 0 from a certain one onward.

15. Other generalizations. The theorem considered in $\$ 13$ dealt with periodic functions, and therefore with Fourier series.

It is natural to pass from periodic functions to almost periodic functions and from Fourier series to Fourier in tegrals (trigonometric integrals); in both cases the number of changes of sign has to be replaced by (a suitable notion of) the density of changes of sign. The first extension was hinted by Norbert Wiener and the present speaker [25] the second by J. D. Tamarkin.

A different kind of generalization was effected by Einar Hille who subjected the role of the Fourier series in the proof of the theorem of $\$ 13$ to a thorough analysis. The most important property of the Fourier series $\sum c_{n} e^{i n x}$ from the point of view of that proof is the following. The operation of differentiation $d / d x$ changes the series in a simple way, it multiplies the coefficient $c_{n}$ by the simple factor in. But the differential operation $\left(1-x^{2}\right)\left(d^{2} / d x^{2}\right)-2 x d / d x$ acts on the development into Legendre polynomials in a similar way. This observation leads to the following theorem, one of numerous analogous results discovered by Hille [15].

Let $N_{n}^{*}$ denote the number of changes of sign of

$$
\left[\left(1-x^{2}\right)\left(d^{2} / d x^{2}\right)-2 x d / d x\right]^{n} f(x)
$$

in the interval $-1 \leqq x \leqq 1$. If $N_{n}^{*}=O(1)$, the function $f(x)$ possesses a development into Legendre polynomials having only a finite number of non-vanishing coefficients, that is, $f(x)$ itself is a polynomial.

16. No derivative changes sign more than a given number of times. The general case. The question raised at the beginning of $\$ 13$ was 
completely answered by A. C. Schaeffer who proved the following theorem. ${ }^{7}$

If, in an open interval $I$, no derivative of $f(x)$ changes sign more than a fixed number of times, $f(x)$ is analytic in $I$.

This theorem contains that of S. Bernstein, discussed in $\$ 9$, since its condition (11) is less restrictive than condition (5). Schaeffer's proof is also very remarkable. It uses tools of the same general character as the proofs of the theorems discussed in $\$ \$ 9-12$, namely inequalities between derivatives, but it adds to those known before an especially original inequality of this kind, another application of which yields a new proof of the theorem of $\$ 13$, and seems to open a new vista on the hypothetical theorem $\mathrm{C}$ of $\$ 7$.

Looking back at the new results and new analogies that have been added quite recently to our knowledge of the subject, we may obtain the impression of grasping the outlines of a well balanced theory leading to further results.

\section{BIBLIOGRAPHY}

1. M. Ålander, Sur le déplacement des zéros des fonctions entières par leur dérivation, dissertation, Upsal, 1914.

2. - Sur les zéros extraordinaires des dérivées des fonctions entières réelles, Arkiv för Mathematik, Astronomi och Fysik vol. 11 (1916) part 15.

3. - Sur les zéros des dérivées des fonctions rationnelles et d'autres fonctions méromorphes, ibid. vol. 14 (1920) part 23.

4. - Sur les dérivêes successives des fonctions régulières, Opuscula Mathematica A. Wiman Dedicata, Lund, 1930, pp. 79-98.

5. S. Bernstein, Sur la définition et les propriêtés des fonctions analytiques d'une variable réelle, Math. Ann. vol. 75 (1914) pp. 449-468.

6. - Leçons sur les propriêtés extrémales et la meilleure approximation des fonctions analytiques d'une variable réelle, Paris, 1926.

7. - On certain properties of regularly monotonic functions (in Russian), Communications de la Société Mathématique de Kharkov (4) vol. 2 (1928) pp. 1-11.

8. - Sur les fonctions régulièrement monotones, C. R. Acad. Sci. Paris vol. 186 (1928) pp. 1266-1269.

9. R. P. Boas, Univalent derivatives of entire function, Duke Math. J. vol. 6 (1940) pp. 719-721.

10. —, Functions with positive derivatives, ibid. vol. 8 (1941) pp. 163-172.

11. - - A note on functions of exponential type, Bull. Amer. Math. Soc. vol. 47 (1941) pp. 750-754.

12. R. P. Boas and G. Polya, Generalizations of completely convex functions, Proc. Nat. Acad. Sci. U.S.A. vol. 27 (1941) pp. 323-325.

${ }^{7}$ Schaeffer $[26,27]$. The question concerning (11) mentioned at the beginning of $\S 13$, was stated by the present speaker at the Stanford University Symposium, August $12,1941$. 
13. - Influence of the signs of the derivatives of a function on its analytic character, Duke Math. J. vol. 9 (1942) pp. 406-424.

14. W. Gontcharoff, Recherches sur les dérivées successives des fonctions analytiques, Ann. Ecole Norm. vol. 47 (1930) pp. 1-78.

15. Einar Hille, On the oscillation of differential transforms. II Characteristic series of boundary value problems, Trans. Amer. Math. Soc. vol. 52 (1942) pp. 463-497.

16. S. Kakeya, An extension of power series, Proceedings of the Physico-Mathematical Society of Japan vol. 14 (1932) pp. 125-138.

17. Norman Levinson, $A$ theorem of Boas, Duke Math. J. vol. 8 (1941) pp. 181182.

18. G. Pòlya, Sur une question concernant les fonctions entières, C. R. Acad. Sci. Paris vol. 158 (1914) pp. 330-333.

19. - Bemerkung zur Theorie der ganzen Funktionen, Jber. Deutschen Math. Verein. vol. 24 (1915) pp. 392-400.

20. — Ueber die Nullstellen sukzessiver Derivierten, Math. Zeit. vol. 12 (1922) pp. 36-60.

21. - Ueber die algebraisch-funktionentheoretischen Untersuchungen von J. L. W. V. Jensen, Kgl. Danske Videnskabernes Selskab. Mathematisk-fysiske Meddelelser, vol. 7 (1927) part 17.

22. - Some problems connected with Fourier's work on transcendental equations, Quart. J. Math. Oxford Ser. vol. 1 (1930) pp. 21-34.

23. - Ueber die Realität der Nullstellen fast aller Ableitungen gewisser ganzer Funktionen, Math. Ann. vol. 114 (1937) pp. 622-634.

24. - On functions whose derivatives do not vanish in a given interval, Proc. Nat. Acad. Sci. U.S.A. vol. 27 (1941) pp. 216-217.

25. G. Polya and Norbert Wiener, On the oscillation of the derivatives of a periodic function, Trans. Amer. Math. Soc. vol. 52 (1942) pp. 249-256.

26. A. C. Schaeffer, Oscillations of the derivatives of a function, Proc. Nat. Acad. Sci. U.S.A vol. 28 (1942) pp. 62-64.

27. - On the oscillation of differential transforms. III, Trans. Amer. Math. Soc. (to appear).

28. I. J. Schoenberg, On the zeros of successive derivatives of integral functions, ibid. vol. 40 (1936) pp. 12-23.

29. G. Szegö, On the oscillation of differential transforms. I, ibid. vol. 52 (1942) pp. $450-462$.

30. S. Takenaka, On the distribution of zero points of the derivatives of an integral transcendental function of order $\rho \leqq 1$, Proc. Imp. Acad. Tokyo vol. 7 (1931) pp. 133-136.

31. - On the expansion of analytic functions in series of analytic functions and its application to the study of the distribution of the zero points of the derivatives of analytic functions, Proceedings of the Physico-Mathematical Society of Japan vol. 13 (1931) pp. 111-132.

32. - On the expansion of analytic functions in generalized Taylor's series, ibid. vol. 14 (1932) pp. 179-196.

33. - On the expansion of integral transcendental functions in generalized Taylor's series, ibid. vol. 14 (1932) pp. 529-542.

34. - On the expansion of an integral transcendental function of the first order in generalized Taylor's series, Proc. Imp. Acad. Tokyo vol. 8 (1932) pp. 59-62.

35. J. M. Whittaker, On Lidstone's series and two-point expansions of analytic functions, Proc. London Math. Soc. (2) vol. 36 (1933) pp. 451-469. 
36. - Interpolatory function theory, Cambridge, 1935.

37. D. V. Widder, Functions whose even derivatives have a prescribed sign, Proc. Nat. Acad. Sci. U.S.A. vol. 26 (1940) pp. 657-659.

38. A. Wiman, Ueber eine asymptotische Eigenschaft der Ableitungen der ganzen Funktionen von den Geschlechtern 1 und 2 mit einer endlichen Anzahl von Nullstellen, Math. Ann. vol. 104 (1930) pp. 169-181.

39. - Ueber die Realität der Nullstellen fast aller Ableitungen gewisser ganzer Funktionen, ibid. vol. 114 (1937) pp. 617-621.

Smith College 